\title{
Effectiveness of Intraoral Chlorhexidine Protocols in the Prevention of Ventilator-Associated Pneumonia: Meta-Analysis and Systematic Review
}

\author{
Cristina C Villar DDS PhD, Claudio M Pannuti DDS PhD, Danielle M Nery DDS, \\ Carlos M R Morillo DDS, Maria José C Carmona MD PhD, and Giuseppe A Romito DDS PhD
}

\begin{abstract}
BACKGROUND: Ventilator-associated pneumonia (VAP) is common in critical patients and related with increased morbidity and mortality. We conducted a systematic review and meta-analysis, with intention-to-treat analysis, of randomized controlled clinical trials that assessed the effectiveness of different intraoral chlorhexidine protocols for the prevention of VAP. METHODS: Search strategies were developed for the MEDLINE, EMBASE, and LILACS databases. MeSH terms were combined with Boolean operators and used to search the databases. Eligible studies were randomized controlled trials of mechanically ventilated subjects receiving oral care with chlorhexidine or standard oral care protocols consisting of or associated with the use of a placebo or no chemicals. Pooled estimates of the relative risk and corresponding 95\% CIs were calculated with random effects models, and heterogeneity was assessed with the Cochran $Q$ statistic and $I^{2}$. RESULTS: The 13 included studies provided data on 1,640 subjects that were randomly allocated to chlorhexidine $(n=834)$ or control $(n=806)$ treatments. A preliminary analysis revealed that oral application of chlorhexidine fails to promote a significant reduction in VAP incidence (relative risk $0.80,95 \%$ CI 0.59-1.07, $\mathrm{I}^{2}=45 \%$ ). However, subgroup analyses showed that chlorhexidine prevents VAP development when used at $2 \%$ concentration (relative risk $0.53,95 \%$ CI $0.31-0.91, I^{2}=0 \%$ ) or 4 times/d (relative risk $0.56,95 \%$ CI $0.38-0.81, \mathrm{I}^{2}=0 \%$ ). CONCLUSIONS: We found that oral care with chlorhexidine is effective in reducing VAP incidence in the adult population if administered at $2 \%$ concentration or 4 times/d. Key words: chlorhexidine; clinical protocols; ventilator-associated pneumonia; meta-analysis; infection; critical care. [Respir Care 2016;61(9):1245-1259. (C) 2016 Daedalus Enterprises]
\end{abstract}

\section{Introduction}

Ventilator-associated pneumonia (VAP) is defined as pneumonia that develops $\geq 48 \mathrm{~h}$ after endotracheal intubation and initiation of mechanical ventilation. ${ }^{1}$ VAP is the second most common nosocomial infection in ICUs and the first most common in patients receiving mechanical ventilation. ${ }^{2}$ The condition is associated with increases

\footnotetext{
Drs Villar, Pannuti, Morillo, and Romito are affiliated with the Discipline of Periodontics, School of Dentistry, and Dr Carmona is affiliated with the Discipline of Anesthesiology, School of Medicine, University of São Paulo-São Paulo, Brazil. Dr Nery is affiliated with the Complexo Hospitalar Municipal de São Bernardo do Campo, São Bernardo do CampoSão Paulo, Brazil.
}

The authors have disclosed no conflicts of interest. in length of hospitalization and ICU stay, morbidity, mortality, and health-care costs. ${ }^{3,4}$ Despite recent advances in diagnosis and treatment of VAP, it continues to be a medical problem of major importance, with an attributable mortality rate between 33 and 50\%.5 Thus, preventive interventions are needed to limit its occurrence.

The development of VAP is related to microbial colonization of the normally sterile lower respiratory tract by microorganisms commonly found in the trachea, orophar-

\footnotetext{
Correspondence: Cristina C Villar DDS PhD, Division of Periodontics, Department of Stomatology, School of Dentistry, University of São Paulo, Avenida Prof. Lineu Prestes, 2227, São Paulo-São Paulo, 05508-000, Brazil. E-mail: villar@usp.br.
}

DOI: $10.4187 /$ respcare. 04610 
ynx, stomach, and small or large intestines. ${ }^{6}$ Although the main route of infection leading to lower respiratory tract infection remains unknown, the primary source of infection for VAP is thought to be the oropharyngeal tract. ${ }^{7}$ Based on this, a significant number of studies have investigated the effect of topical oral antiseptics in VAP prevention. Among these antiseptics, chlorhexidine gluconate has attracted considerable attention, as evidenced by numerous randomized controlled clinical trials that have investigated the effect of oral chlorhexidine use in VAP prevention. ${ }^{8-25}$

Results from the aforementioned randomized controlled trials and meta-analyses ${ }^{26-30}$ that analyzed the effect of oral care with chlorhexidine on VAP prevention are conflicting. Discrepant findings may have resulted from differences in study populations, diagnostic criteria for VAP, chlorhexidine concentration, and frequency of use. Metaanalyses have not reported the impact of specific protocols of oral care with chlorhexidine on VAP prevention. Moreover, previous meta-analysis mixed together outcomes reported on intention-to-treat and per-protocol basis. Therefore, in this paper, a systematic review and meta-analysis, with intention-to-treat analysis, of randomized controlled clinical trials was conducted to determine the effectiveness of oral decontamination with chlorhexidine and to compare specific protocols of oral care with chlorhexidine in VAP prevention.

\section{Methods}

\section{Focused Question}

We conducted a systematic review of the literature to assess the following focused PICO (patient or population, intervention, control or comparator, and outcome) question: In subjects endotracheally intubated and mechanically ventilated, does oral decontamination with chlorhexidine prevent the development of VAP, when compared with placebo or standard care or no treatment? As a second aim, this systematic review assessed the question: Which dose, frequency, or mode of use provides the best effect in the prevention of VAP? This systematic review was reported according to the PRISMA statement guidelines. ${ }^{31}$

\section{Eligibility Criteria}

Type of Studies. Only randomized controlled trials that reported data using an intention-to-treat approach or provided enough information that per-protocol results could be adjusted into an intention-to-treat format were eligible for this review.

Study Population. The population of interest included intubated subjects receiving mechanical ventilation.

\section{QUICK LOOK}

\section{Current knowledge}

In recent years, many regimens of oral care with chlorhexidine have been used on mechanically ventilated patients to prevent the development of ventilator-associated pneumonia (VAP). However, results from randomized controlled trials and meta-analysis that analyzed the effect of oral care with chlorhexidine on VAP prevention are still conflicting.

\section{What this paper contributes to our knowledge}

Results from this systematic review and meta-analysis indicate that oral care with chlorhexidine is effective in reducing VAP incidence only in the adult population and if administered at a $2 \%$ concentration or 4 times/d.

Type of Intervention and Comparison. Oral decontamination protocols using chlorhexidine (test group) were compared with standard oral care protocols consisting of or associated with the use of (1) a placebo or (2) no treatment.

Outcome Measures. The primary outcome was incidence of VAP, reported as the number/percentage of affected subjects.

\section{Search Strategy}

Search strategies were developed for the MEDLINE, EMBASE, and LILACS databases. MeSH terms and key words were combined with Boolean operators and used to search the databases. All searches were done without language restriction, up to January 2015. The following terms were used: ([chlorhexidine OR "gluconate chlorhexidine" OR "oral decontamination" OR "oral hygiene" OR antiseptics OR "antiseptic decontamination"] AND ["ventilator-associated pneumonia" OR VAP OR "nosocomial pneumonia" OR pneumonia OR intubation OR "mechanical ventilation" OR "intensive care units" OR “critical care"]) AND ("clinical trial" OR RCT OR "randomized controlled trial" OR "randomized controlled clinical trial"). Electronic search was complemented by manual searches of the reference lists of selected full articles.

\section{Exclusion Criteria}

Reviews, in vitro and animal studies, case reports, observational studies, and studies without control groups were not included. 


\section{Screening Methods and Data Extraction}

Two calibrated reviewers (DMN and CCV) independently screened titles and abstracts. Studies appearing to meet the inclusion criteria or those with insufficient information in the title and abstract to make a clear decision, were selected for full manuscript evaluation, which was carried out independently by the same 2 reviewers to determine study eligibility. Any disagreement was solved by discussion with a third reviewer (CMP). Reference lists of previous reviews and included studies were hand-searched. Studies that met the inclusion criteria underwent a validity assessment. Reasons for rejecting studies were recorded. Agreement between reviewers was described by kappa coefficient. Data were extracted independently by the same reviewers.

The following data were extracted and recorded: citation, setting and location of the trial, characteristics of participants, characteristics of the intervention (concentration, dose, frequency, and type of application), sample size, definition of VAP, and length of follow-up.

\section{Quality Assessment and Data Synthesis}

Quality assessment of the included studies was performed independently by 2 reviewers (DMN and CCV), with disagreements resolved by a third adjudicator (CMP). The following 6 domains were assessed as having low risk, high risk, or unclear risk of bias, according to the Cochrane Collaboration's tool for assessing risk of bias. ${ }^{32}$ Then studies were categorized as follows: (1) low risk of bias, if all domains were met; (2) unclear risk of bias, if one or more domains were classified as having unclear risk of bias; and (3) "high risk" of bias, if one or more domains were not met.

\section{Data Analysis}

Analyses were performed using Review Manager 5.3 software (Cochrane Information Management System). Data on the incidence of VAP was extracted as dichotomous variables. Pooled estimates of the relative risk and the corresponding 95\% CI were calculated using random effects models. Subgroup statistical heterogeneity among the studies was assessed with the Cochran $\mathrm{Q}$ statistic and $\mathrm{I}^{2}$.

\section{Results}

The computerized search strategy yielded 211 citations, of which 53 were screened for potentially meeting the inclusion criteria (Fig. 1). Independent screening of abstracts led to the rejection of 30 articles (Fig. 1). The full text of the remaining 23 publications was obtained for

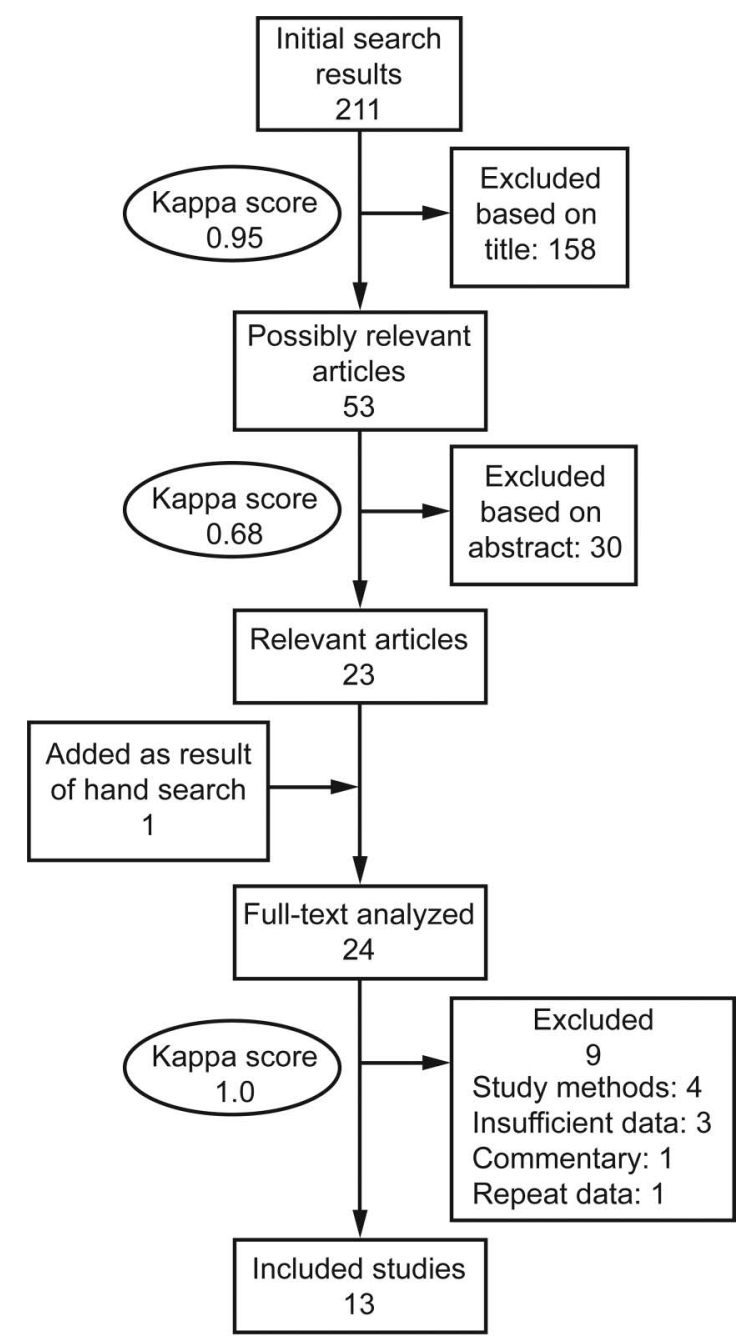

Fig. 1. Flow chart.

review and possible inclusion. Scanning of reference lists yielded one additional study (Fig. 1). Of the 24 publications preselected, 9 articles were further excluded for reasons indicated in Figure 1. As a result, 13 studies published in English between September 2000 and November 2012 were included in this meta-analysis. The characteristics of the final trials retained are reported in Table 1.

\section{Subject Selection and Characteristics}

The 13 included studies provided data on 1,640 subjects who were randomly allocated to chlorhexidine $(n=834)$ or control $(n=806)$ treatments. Studies enrolled subjects expected to require orotracheal or nasotracheal intubation and mechanical ventilation. 8-10,12,14,15,17-19,21,22,24,25 Among these, some studies required mechanical ventilation for at least 48 h. ${ }^{10,12,22,24}$ Other studies only included subjects with medical conditions suggesting an ICU stay of $\geq 48 \mathrm{~h},{ }^{15}$ $3 \mathrm{~d},{ }^{18}$ or $5 \mathrm{~d}^{8,9}$ (Table 1). In 3 studies, research subjects 


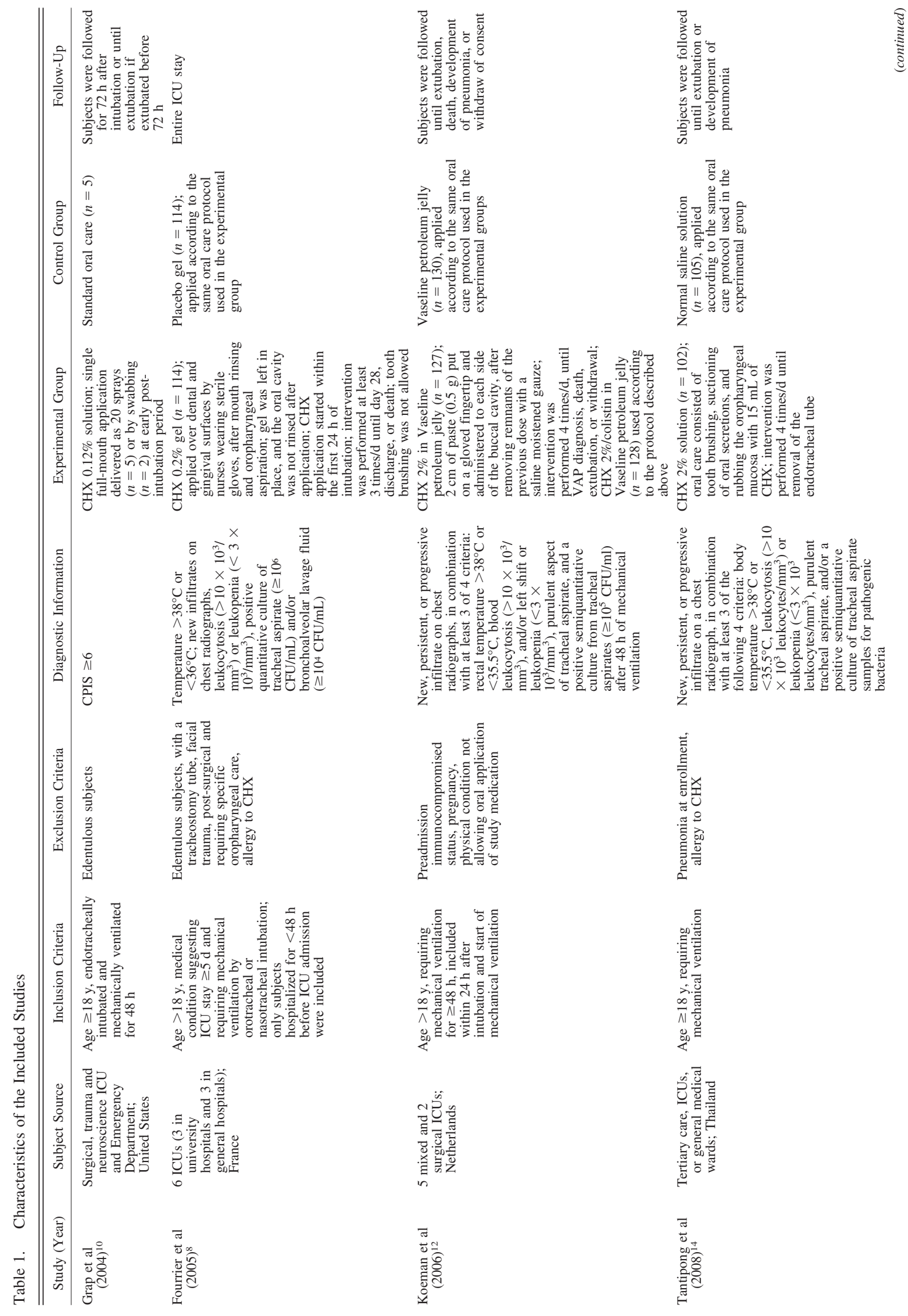




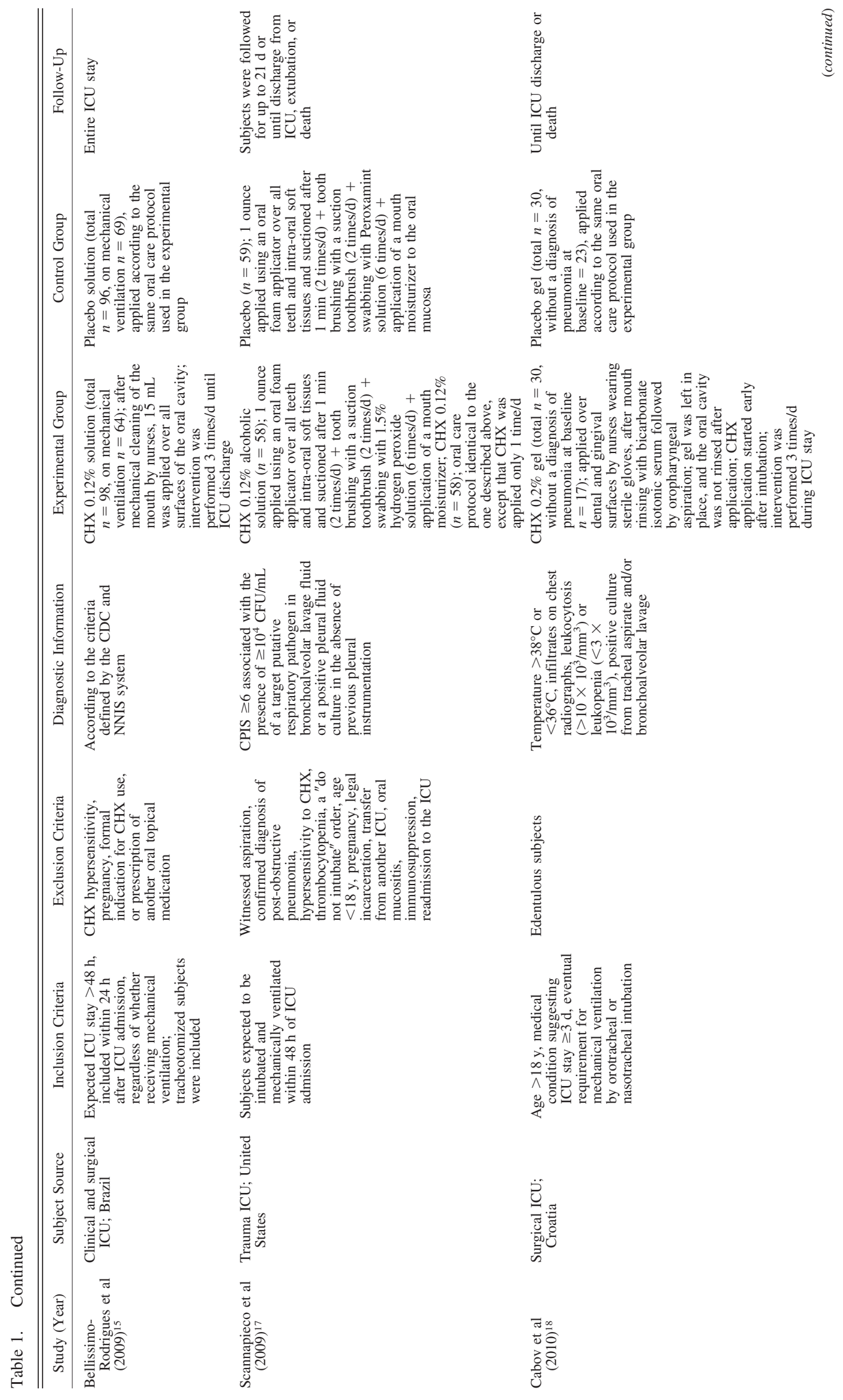




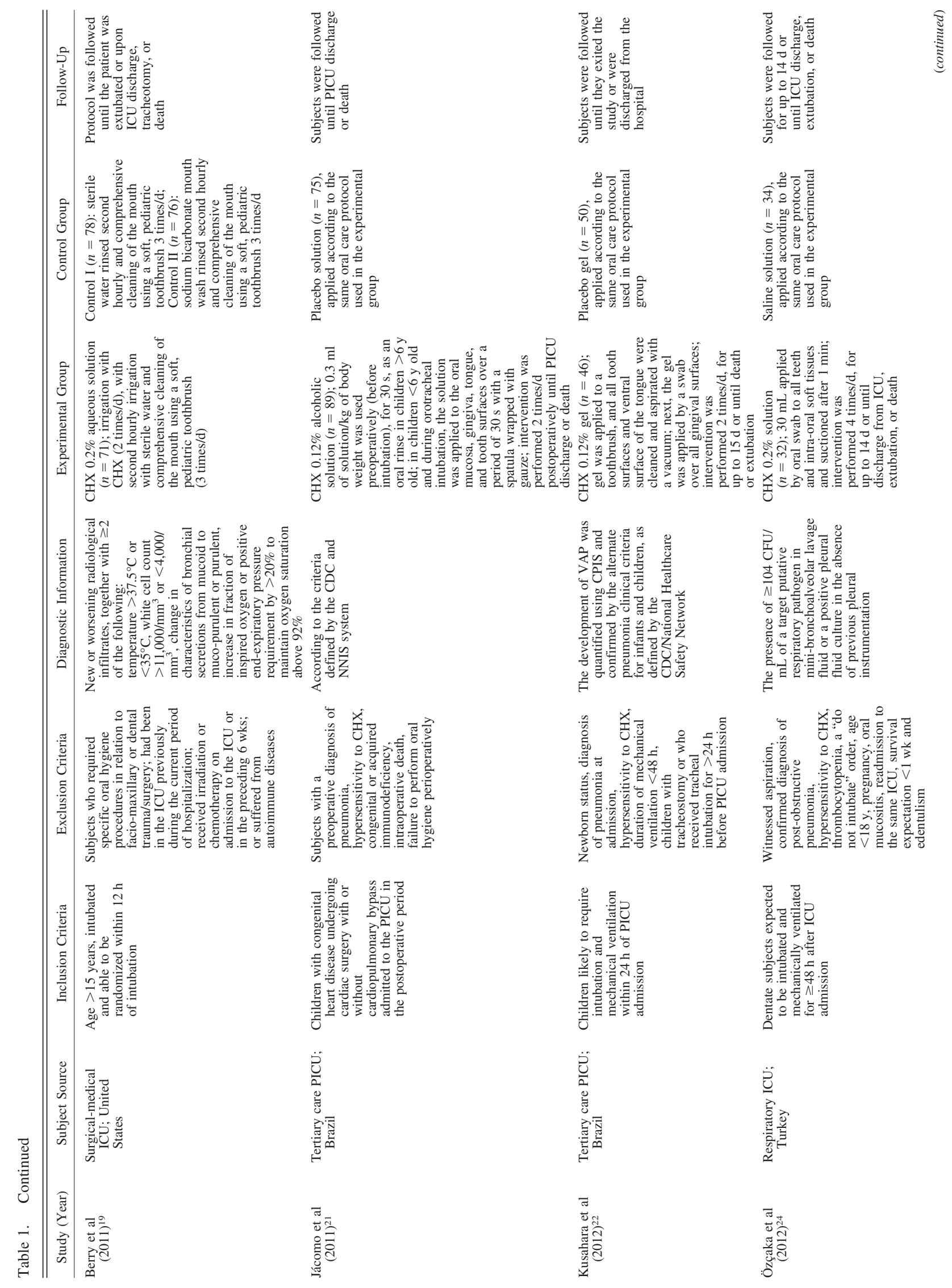




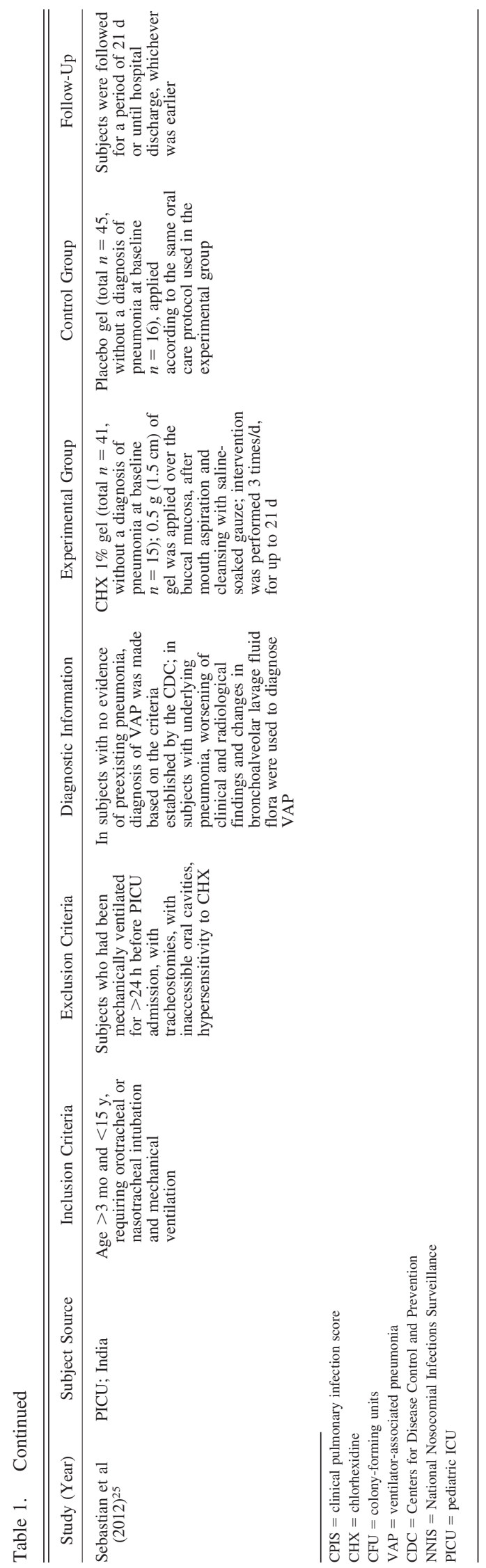

were children. ${ }^{21,22,25}$ All of the remaining studies included subjects age $>15 \mathrm{y}^{19}$ or $\geq 18 \mathrm{y}^{8,9,10,12,14,18}$ (see Table 1).

Trials were set in various ICUs and emergency services. Most studies included subjects from clinical surgical ICUs ${ }^{8,9,15,18,19}$ and pediatric ICUs. ${ }^{21,22,25}$ Two studies included subjects admitted to trauma ICUs. ${ }^{10,17}$ Moreover, single trials were carried out in neuroscience ICU, ${ }^{10}$ mixed ICU, ${ }^{8}$ emergency department, ${ }^{10}$ and general medical wards $^{14}$ (Table 1). Average sample sizes, inclusion and exclusion criteria, and VAP diagnostic methods and criteria varied considerably among studies (Table 1).

\section{Oral Care With Chlorhexidine}

Included studies were also quite heterogeneous in their intervention regimens. Among them, chlorhexidine was used at concentrations of $0.12 \%,{ }^{10,15,17,21,22} 0.2 \%, 8,9,18,19,24$ $1 \%, 25$ and $2 \%{ }^{12,14}$ (Table 1). Chlorhexidine was applied as oral rinse solution, ${ }^{10,14,15,19,21,24}$ gel, $, 8,9,18,22,25$ Vaseline petroleum jelly, ${ }^{12}$ and foam ${ }^{17}$ (Table 1 ). When specified by the authors, chlorhexidine solutions were reported as aqueous $^{19}$ or alcoholic. ${ }^{17,21}$

The frequency of chlorhexidine oral application also varied among the studies. Chlorhexidine was used in a single dose at intubation, ${ }^{10}$ once/d, ${ }^{17}$ twice/d, ${ }^{17,19,21,22} 3$ times/d, ${ }^{8,9,15,18,25}$ or 4 times/d ${ }^{12,14,24}$ (Table 1).

\section{Methodological Quality of the Studies}

Studies' individual risk of bias were assessed and listed in Table 2. Details related to the method of randomization were provided in all studies. ${ }^{8-10,12,14,15,17-19,21,22,24,25}$ Allocation concealment was adequately described only in 4 studies. ${ }^{8,15,17,21}$ Moreover, one study ${ }^{25}$ reported that allocation was concealed but did not provide details of the concealment. The remaining 8 studies did not provide any information about allocation concealment.9,10,12,14,18,19,22,24 Whereas study subjects and personnel were blinded in only 8 trials, $8,12,15,17,18,21,22,25$ outcome assessors were blinded in all studies. $8-10,12,14,15,17-19,21,22,24,25$

Incomplete outcome data were adequately addressed in 5 studies. ${ }^{12,15,21,24,25}$ In 3 studies, ${ }^{9,17,18}$ the reasons for missing data in each group were not provided. In 2 others, the dropout rate was significant higher in the chlorhexidine group. ${ }^{10,19}$ In Fourrier et al, ${ }^{9}$ the proportion of missing outcomes compared with observed event risk was high enough to induce relevant bias. Finally, the reasons for missing outcomes were likely to be related to the true outcome in Kusahara et al. ${ }^{22}$

Sample size calculation was not described in 4 studies. $9,10,18,22$ Moreover, in the other 4, final sample size was smaller than the number indicated by sample size calculations. ${ }^{8,14,19,25}$ 


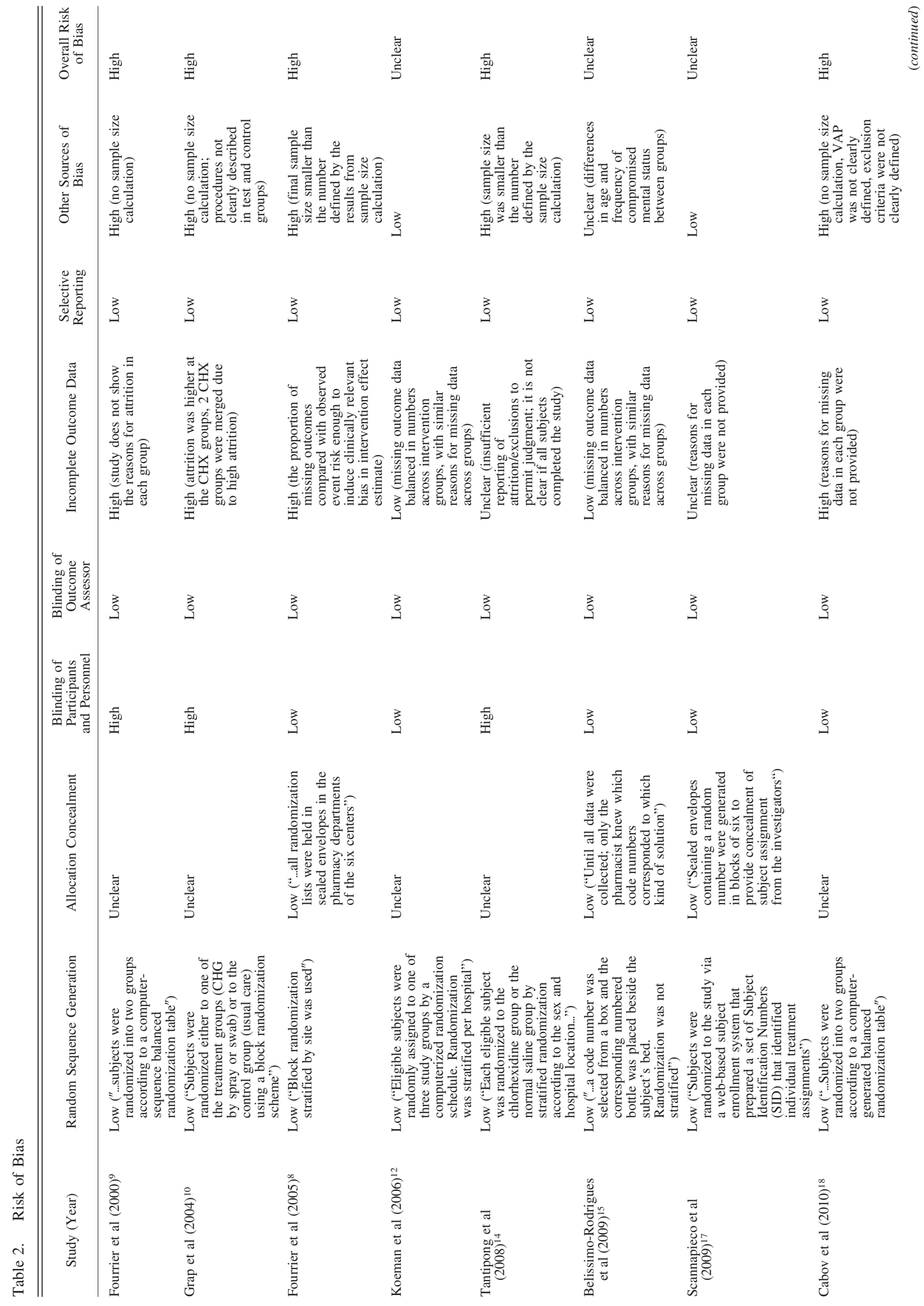




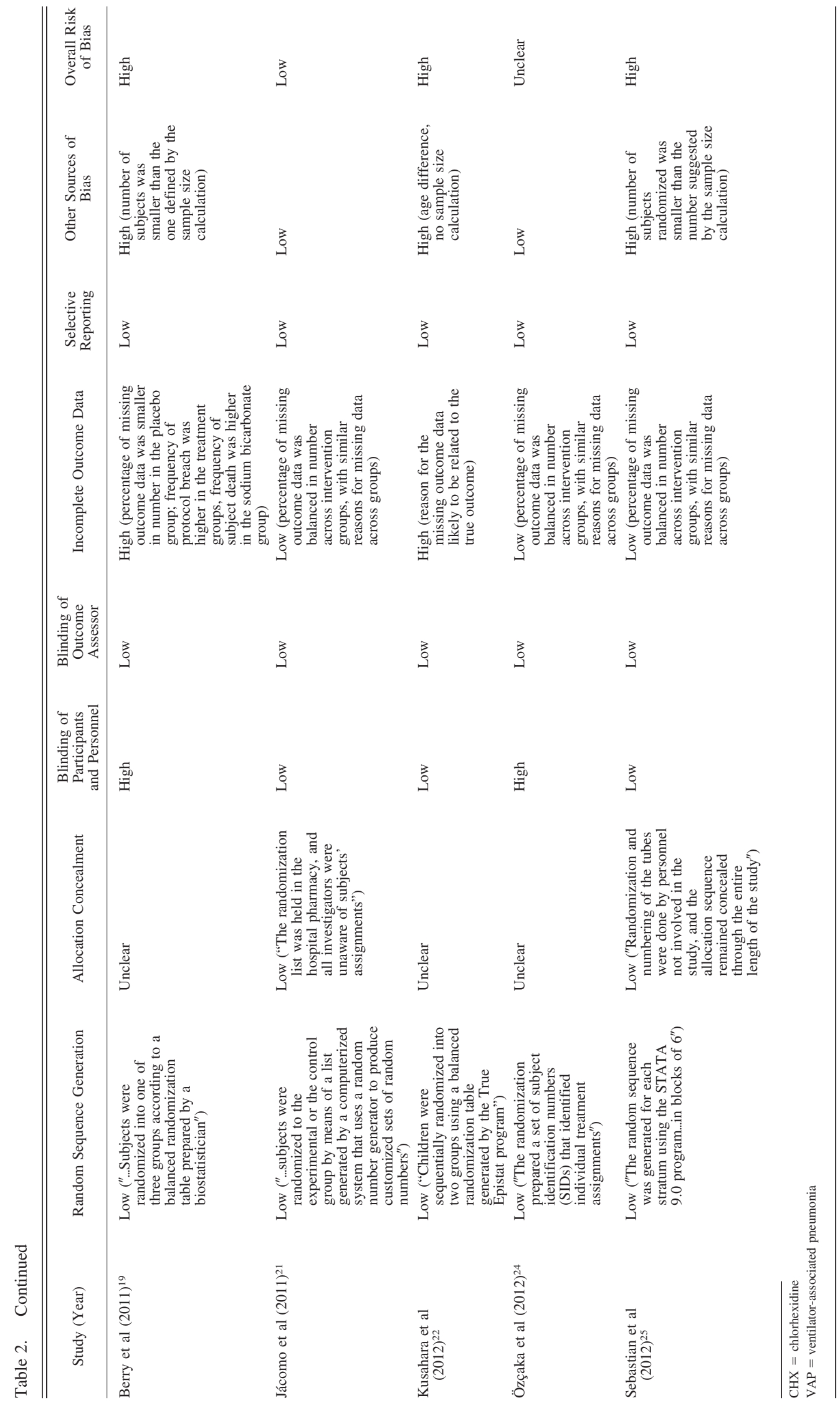




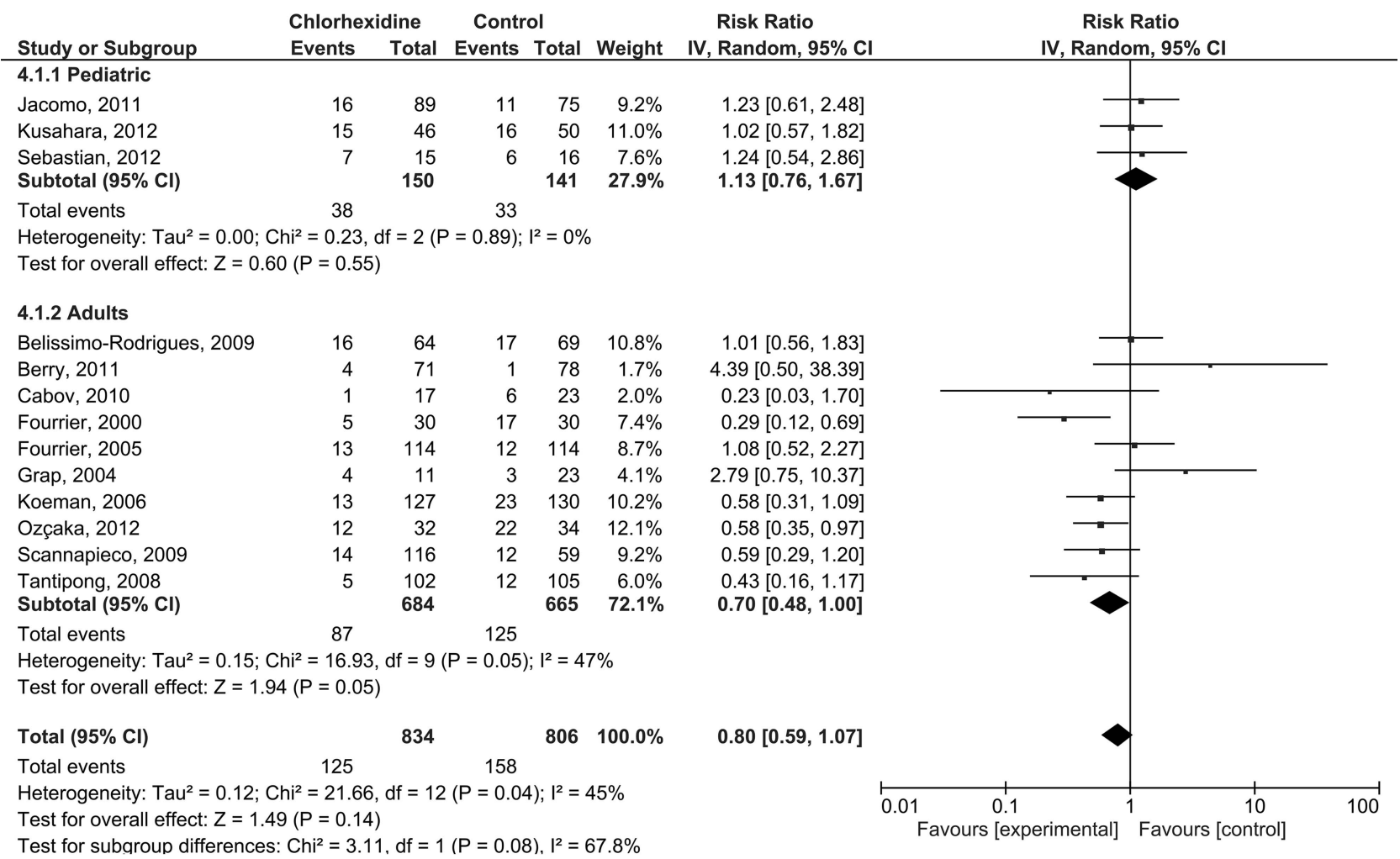

Fig. 2. Effect of oral care with chlorhexidine on ventilator-associated pneumonia prevention.

\section{Effect of Oral Care With Chlorhexidine on VAP Prevention}

A preliminary analysis including 1,640 pediatric and adult subjects revealed that oral application of chlorhexidine did not promote a significant reduction in VAP incidence (relative risk $0.80,95 \%$ CI $0.59-1.07, \mathrm{I}^{2}=45 \%$ ) (Fig. 2). Next, subgroup analyses were conducted to compare the effect of chlorhexidine in pediatric and adult populations. Similar to the results found in the overall study population, oral care with chlorhexidine failed to prevent VAP in the pediatric population (relative risk $1.13,95 \% \mathrm{CI}$ $0.76-1.67, \mathrm{I}^{2}=0 \%$ ) (see Fig. 2). Nonetheless, oral application of chlorhexidine promoted a trend toward a protective effect in adult subjects (relative risk 0.70, 95\% CI $0.48-1.00, \mathrm{I}^{2}=47 \%$ ) (see Fig. 2). Due to the limited number of studies that investigated the effect of oral care with chlorhexidine on VAP prevention in pediatric subjects and the lack of effects of oral care with chlorhexidine in this study population, the following subgroup analyses were conducted based on adult population data only.

\section{Effect of Chlorhexidine Concentration}

Subgroup analysis investigated chlorhexidine used in concentrations of $0.12,0.2$, and $2 \%$ (Fig. 3). At the lowest concentrations tested $(0.12$ and $0.2 \%)$, chlorhexidine failed to prevent VAP development $(0.12 \%$ chlorhexidine: relative risk $1.00,95 \%$ CI $0.51-1.99, \mathrm{I}^{2}=54 \%$; $0.2 \%$ chlorhexidine: relative risk $0.63,95 \%$ CI $0.32-1.22, \mathrm{I}^{2}=57 \%$ ). In sharp contrast, $2 \%$ chlorhexidine promoted a significant reduction in VAP incidence (relative risk $0.53,95 \%$ CI $\left.0.31-0.91, \mathrm{I}^{2}=0 \%\right)$.

\section{Effect of Chlorhexidine Frequency of Use}

Subgroup analyses investigated chlorhexidine used in a single application at intubation and once, twice, 3 times, or 4 times daily (Fig. 4). When used as a single application dose at intubation, in the study published by Grap et $a l,{ }^{10}$ chlorhexidine failed to reduce the incidence of VAP (relative risk 2.79, 95\% CI 0.75-10.37). Likewise, chlorhexidine used at frequencies of once/d, twice/d, and 3 times/d also failed to prevent VAP development (once/d: relative risk 0.59 , 95\% CI 0.25 1.40; twice/d: relative risk $1.25,95 \%$ CI $0.19-8.31$, $\mathrm{I}^{2}=65 \% ; 3$ times/d: relative risk $0.64,95 \%$ CI $0.31-$ $1.31, \mathrm{I}^{2}=62 \%$ ). The protective effect of chlorhexidine was only achieved when its frequency of use was increased to 4 times/d (relative risk 0.56 , $95 \%$ CI $0.38-$ $\left.0.81, \mathrm{I}^{2}=0 \%\right)$. 


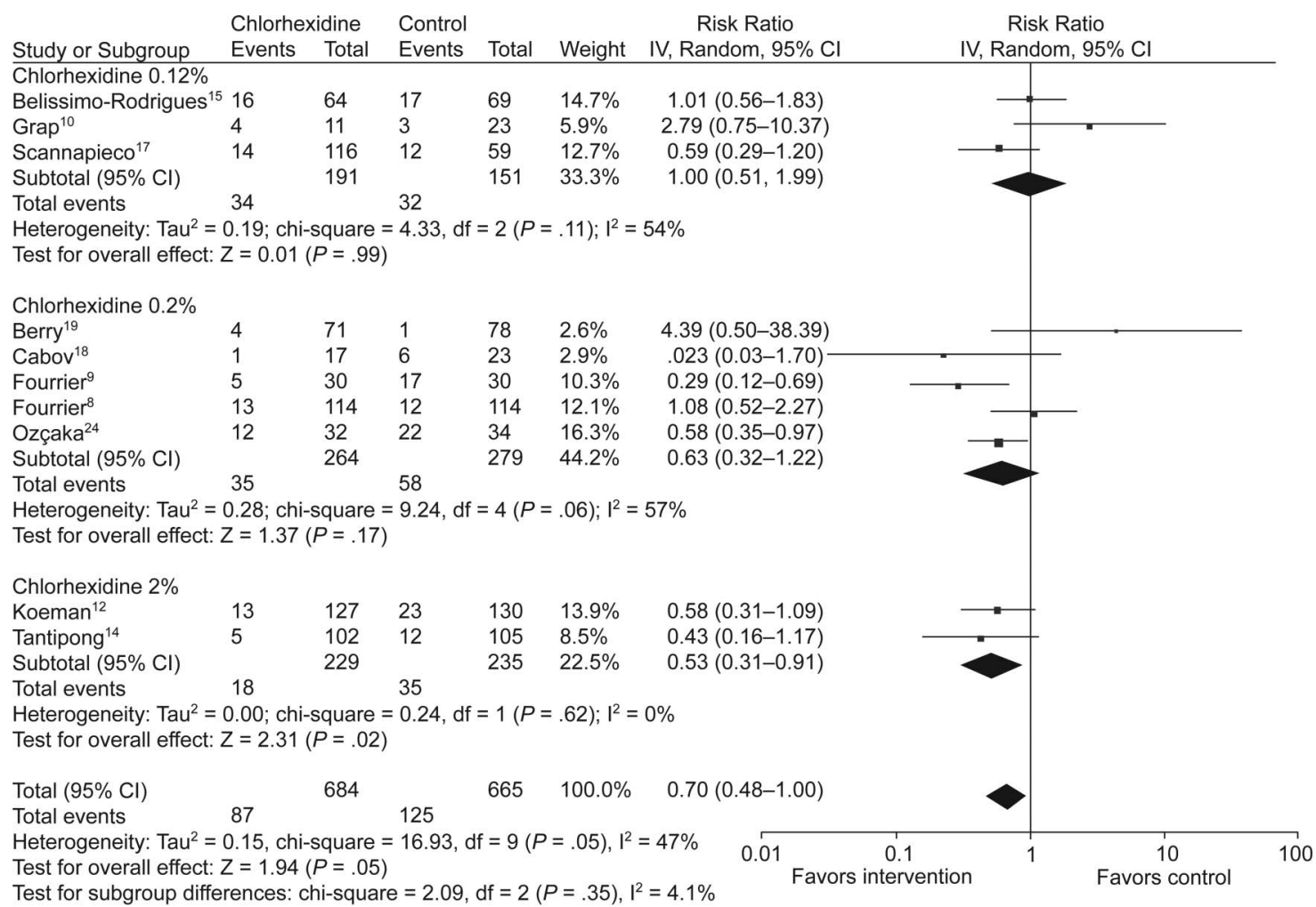

Fig. 3. Effect of chlorhexidine concentration on ventilator-associated pneumonia prevention.

\section{Effect of Chlorhexidine Used as Monotherapy or in Combination With Mechanical Means}

In some studies, chlorhexidine was the only form of oral care. ${ }^{8-10,12,18,24}$ On the contrary, in some others, chlorhexidine was associated with mechanical debridement. ${ }^{14,15,17,19}$ Therefore, an analysis was undertaken to assess the effectiveness of chlorhexidine used alone and in association with mechanical means for the prevention of VAP (Fig. 5). Used as monotherapy, chlorhexidine failed to reduce VAP incidence (relative risk $0.65,95 \%$ CI $0.39-1.09, \mathrm{I}^{2}=$ $55 \%$ ). Similarly, chlorhexidine did not promote a significant reduction in VAP incidence when used in conjunction with mechanical cleansing of the oral cavity (relative risk $0.77,95 \%$ CI $0.43-1.39, \mathrm{I}^{2}=42 \%$ ).

\section{Safety}

Six studies reported that the oral use of chlorhexidine was associated with no adverse effects. ${ }^{15,17,19,21,24,25}$ Another 6 studies failed to provide information about its safety. ${ }^{8-10,12,18,22}$ One study reported that mild and reversible irritation of the oral mucosa was more common in subjects treated with chlorhexidine $2 \%$ solution than in those treated with normal saline. ${ }^{14}$
Discussion

With an intention-to-treat analysis, the present metaanalysis provides the most comprehensive assessment to date of the effect of different protocols of oral care with chlorhexidine in VAP prevention in a non-cardiac surgery population. According to our results, the effectiveness of oral care with chlorhexidine in VAP prevention is influenced by the age of the population and the concentration and frequency of application of chlorhexidine.

The current study demonstrated that oral care with chlorhexidine promoted a trend toward VAP prevention in adult subjects but failed to prevent disease development in newborns and infants. There are 3 possible explanations for this discrepancy. First, it is plausible that the antimicrobial effects of chlorhexidine cannot overcome the relative immaturity of the immune system of newborns and infants. The relevance of newborn respiratory innate immunity to the pathogenesis of respiratory diseases in newborns and infants is beginning to surface. Plasmatic levels of complement components and other multifunctional soluble immune proteins are significantly lower in newborns compared with adults. ${ }^{33}$ Moreover, existing evidence based on animal models indicates that a post-natal impairment of TLR2 and TLR4 expression negatively affects inflammatory responses following intratracheal administration of 


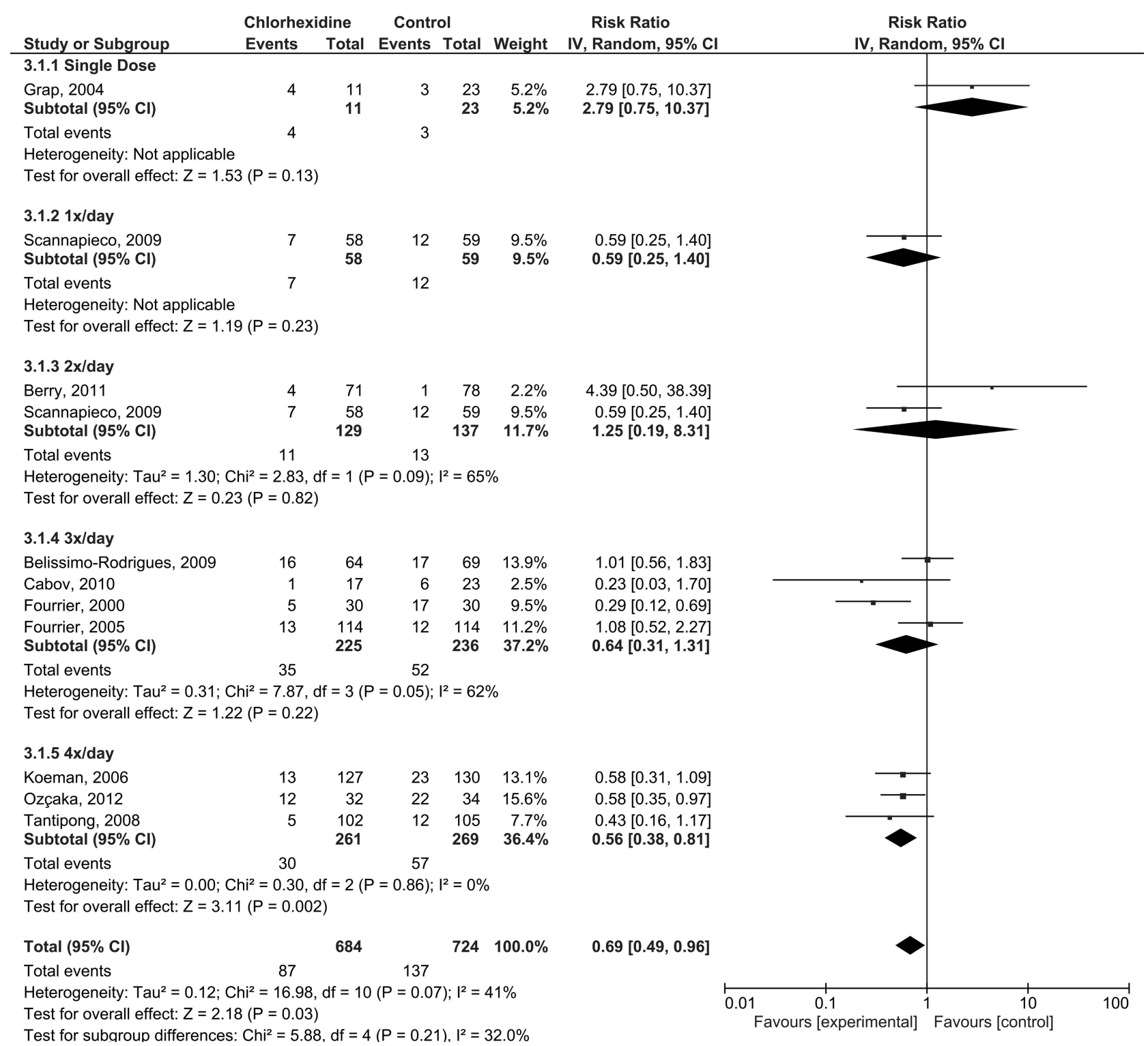

Fig. 4. Effect of chlorhexidine frequency of use on ventilator-associated pneumonia prevention.

Gram-negative bacteria early in life. ${ }^{34}$ Likewise, a strong bias against $\mathrm{T}$ helper cell type 1 polarization of the immune response is also thought to make infants more susceptible to microbial infections. ${ }^{35}$ Second, it is reasonable to speculate that the small oral cavity associated with the relatively large tongue in newborns and infants is likely to pose technical difficulties in providing proper oral care with chlorhexidine to these subjects. Last, the lack of chlorhexidine effect in the pediatric population might be simply explained by the fact that none of the pediatric trials used $2 \%$ formulations or rendered oral decontamination with chlorhexidine 4 times/d.

This meta-analysis demonstrated that the effectiveness of oral care with chlorhexidine on prevention of VAP is dose- and frequency-dependent. Subgroup analysis dem- onstrated that 0.12 and $0.2 \%$ chlorhexidine failed to promote a significant reduction in VAP incidence in adult subjects. In sharp contrast, $2 \%$ chlorhexidine promoted a significant reduction in the incidence of VAP, with a relative risk of 0.53 . Two previous meta-analyses showed similar results, with a relative risk of 0.53 for chlorhexidine $2 \% .28,36$ The antibacterial activity of chlorhexidine is dose-dependent. ${ }^{37,38}$ Higher and longer lasting antimicrobial activity has been reported for $2 \%$ chlorhexidine as compared with less concentrated formulations, ${ }^{39}$ which could explain the superior results of oral care with $2 \%$ chlorhexidine in VAP prevention. Nonetheless, it is important to note that data about the tolerance of $2 \%$ solutions were provided by only one study that reported mild and reversible irritation of the oral mucosa with the use of 


\section{InTRaOral ChlorheXidine for Prevention of VAP}

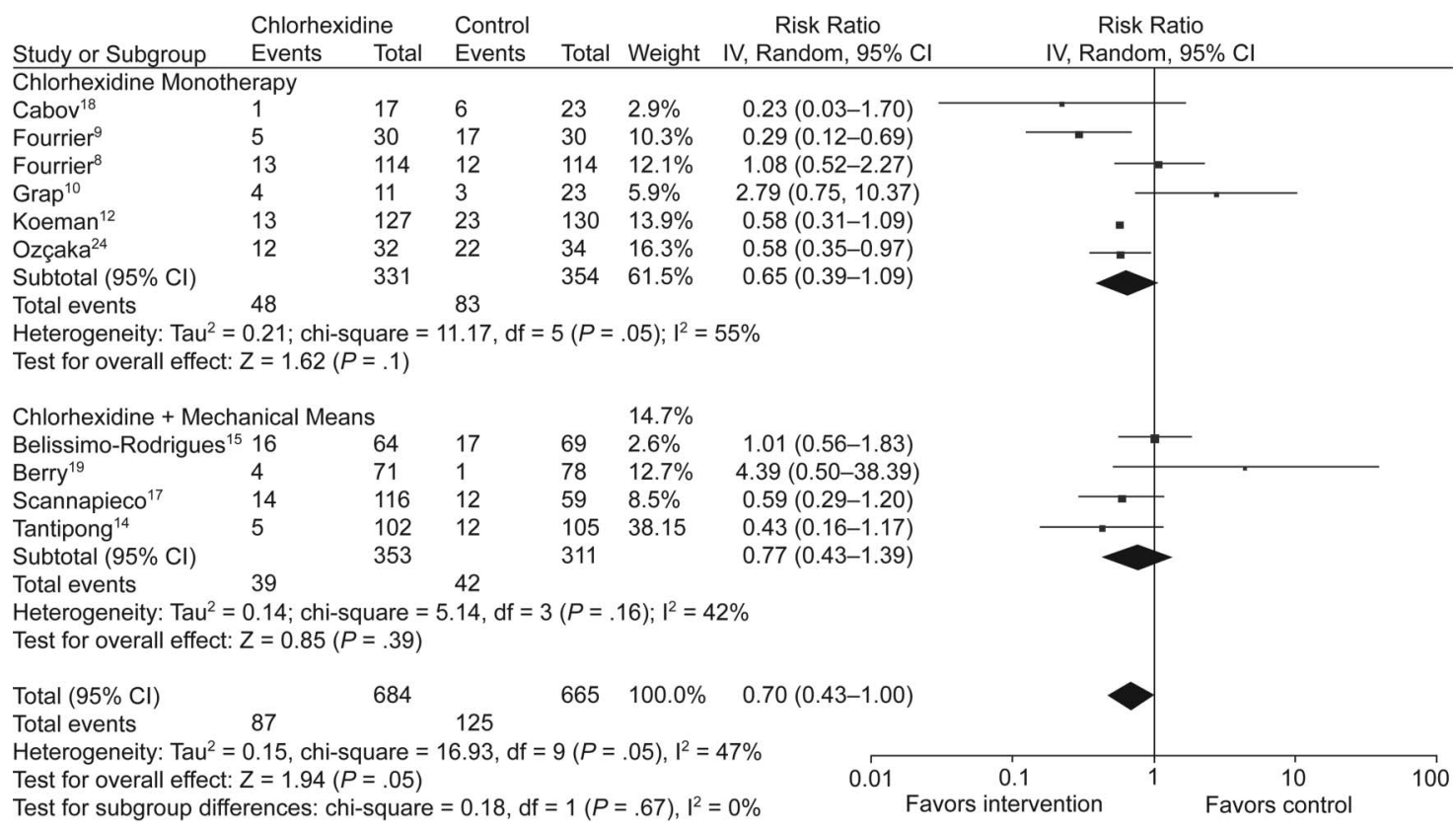

Fig. 5. Effect of chlorhexidine used as monotherapy or in combination with mechanical means on ventilator-associated pneumonia prevention.

$2 \%$ chlorhexidine solution. ${ }^{14}$ Moreover, $2 \%$ chlorhexidine solutions are not available worldwide and are often only made for study purposes.

This study showed for the first time that oral care with chlorhexidine is only effective in reducing VAP incidence when provided 4 times/d. Numerous authors have demonstrated the immediate antibacterial effect of chlorhexidine and the persistence of its substantivity for up to 12-14 h after its administration. However, the clinical relevance of this information has been challenged, since rising evidence suggests that although chlorhexidine can be found in the oral cavity for $>12 \mathrm{~h}$, its antimicrobial activity lasts only $7 \mathrm{~h}$ after a mouth rinse. ${ }^{37,38}$ Thus, it is likely that the effectiveness of oral care with chlorhexidine in VAP prevention is dependent on its persistent antimicrobial activity.

Along these lines, $2 \%$ chlorhexidine was used in only 2 of the total of 10 adult population trials included in this meta-analysis. On a patient level, this signifies that only $33 \%$ of subjects receiving oral care with chlorhexidine were treated with the $2 \%$ formulation. Likewise, chlorhexidine was administered 4 times/d in only 3 trials included in this meta-analysis, encompassing only $38 \%$ of subjects receiving oral care with chlorhexidine. As previously mentioned, the current study showed that oral care with chlorhexidine promoted only a trend toward VAP prevention in adult subjects. Thus, it is reasonable to speculate that the overall effect of oral care with chlorhexidine in VAP prevention could have been stronger if more trials had administered chlorhexidine at $2 \%$ or rendered treatment 4 times/d. Also, the wide variety of combinations of chlorhexidine concentrations and dose intervals reported in studies included in this meta-analysis have precluded the investigation of potential interplays of chlorhexidine concentration and frequency of use in VAP prevention. A ventilator bundle is a group of interventions related to ventilator care that, when implemented together, promotes significantly better outcomes. The VAP prevention bundle is a widely used ICU protocol that includes elevation of the head of the bed, daily sedation vacations and assessment of readiness to extubate, peptic ulcer disease prophylaxis, deep vein thrombosis prophylaxis, and oral decontamination with chlorhexidine. Thus, it is also plausible that oral decontamination with chlorhexidine failed to promote an overall significant reduction in VAP incidence because other bundle prevention measures had been successfully implemented and limited VAP development.

Subanalyses conducted to specifically assess the effectiveness of oral chlorhexidine used alone and in association with mechanical means in the prevention of VAP showed that none of these protocols were able to reduce VAP incidence. These results, however, must be interpreted cautiously due to the large methodological heterogeneity across the limited number of studies included in this subanalysis. The observation that oral chlorhexidine alone might be slightly superior due to its association with mechanical means for VAP prevention is likely to be misleading. First, no direct comparison was made between these protocols. Second, the meta-analysis that assessed the efficacy of chlorhexidine associated with mechanical means included fewer subjects receiving $2 \%$ chlorhexidine and/or rendered treatment 4 times/d than the meta-analysis 
of studies assessing the efficacy of chlorhexidine alone. Of interest, a meta-analysis of 4 low-quality trials found no difference between oral care with chlorhexidine plus tooth brushing and oral care with chlorhexidine alone in terms of VAP prevention. ${ }^{28}$

Finally, only 2 studies included in this meta-analysis reported the periodontal conditions of enrolled subjects. ${ }^{17,24}$ Potential associations between periodontal disease and periodontal disease-associated micro-organisms and the development of nosocomial pneumonia have been already proposed. ${ }^{40}$ Thus, it is plausible to speculate that oral care with chlorhexidine is more likely to prevent VAP development in subjects with periodontal infection.

\section{Conclusions}

We found that oral care with chlorhexidine is effective in reducing VAP incidence in the adult population only if chlorhexidine is administered at $2 \%$ or 4 times/d. These findings, however, must be interpreted cautiously, due to the high heterogeneity of the studies and small number of trials that tested the safety and effectiveness of chlorhexidine at $2 \%$ or rendered treatment 4 times/d. Further investigation of intervention protocols implementing oral chlorhexidine at high concentration and frequency to reduce VAP in subjects with a known periodontal status is required before definitive recommendations can be made.

\section{REFERENCES}

1. Tablan OC, Anderson LJ, Besser R, Bridges C, Hajjeh R. Guidelines for preventing health-care-associated pneumonia, 2003: recommendations of CDC and the Healthcare Infection Control Practices Advisory Committee. MMWR Recomm Rep 2004;53(RR-3):1-36.

2. Safdar N, Crnich CJ, Maki DG. The pathogenesis of ventilator-associated pneumonia: its relevance to developing effective strategies for prevention. Respir Care 2005;50(6):725-739; discussion 739-741.

3. Vincent JL, Rello J, Marshall J, Silva E, Anzueto A, Martin CD, et al. International study of the prevalence and outcomes of infection in intensive care units. JAMA 2009;302(21):2323-2329.

4. Melsen WG, Rovers MM, Groenwold RH, Bergmans DC, Camus C, Bauer TT, et al. Attributable mortality of ventilator-associated pneumonia: a meta-analysis of individual patient data from randomised prevention studies. Lancet Infect Dis 2013;13(8):665-671.

5. Kalanuria AA, Zai W, Mirski M. Ventilator-associated pneumonia in the ICU. Critical Care 2014;18(2):208.

6. Inglis TJ. New insights into the pathogenesis of ventilator-associated pneumonia. J Hosp Infect 1995;30(Suppl):409-413.

7. Bergmans D, Bonten M. Healthcare-associated Pneumonia (Chapter 22) in Mayhall CG. Hospital Epidemiology and Infection Control. 4th edition. Philadelphia, Pennsylvania: Lippincott Williams and Wilkins; 2011:311-316.

8. Fourrier F, Dubois D, Pronnier P, Herbecq P, Leroy O, Desmettre T, et al. Effect of gingival and dental plaque antiseptic decontamination on nosocomial infections acquired in the intensive care unit: a double-blind placebo-controlled multicenter study. Crit Care Med 2005; 33(8):1728-1735.

9. Fourrier F, Cau-Pottier E, Boutigny H, Roussel-Delvallez M, Jourdain M, Chopin C. Effects of dental plaque antiseptic decontamina- tion on bacterial colonization and nosocomial infections in critically ill patients. Intensive Care Med 2000;26(9):1239-1247.

10. Grap MJ, Munro CL, Elswick RK Jr., Sessler CN, Ward KR. Duration of action of a single, early oral application of chlorhexidine on oral microbial flora in mechanically ventilated patients: a pilot study. Heart Lung 2004;33(2):83-91.

11. Bopp M, Darby M, Loftin KC, Broscious S. Effects of daily oral care with $0.12 \%$ chlorhexidine gluconate and a standard oral care protocol on the development of nosocomial pneumonia in intubated patients: a pilot study. J Dent Hyg 2006;80(3):9.

12. Koeman M, van der Ven AJ, Hak E, Joore HC, Kaasjager K, de Smet $\mathrm{AG}$, et al. Oral decontamination with chlorhexidine reduces the incidence of ventilator-associated pneumonia. Am J Respir Crit Care Med 2006;173(12):1348-1355.

13. Koeman M, van der Ven AJ, Hak E, Joore JC, Kaasjager HA, de Smet AM, et al. Less ventilator-associated pneumonia after oral decontamination with chlorhexidine: a randomised trial. Ned Tijdschr Geneeskd 2008;152(13):752-759.

14. Tantipong H, Morkchareonpong C, Jaiyindee S, Thamlikitkul V. Randomized controlled trial and meta-analysis of oral decontamination with $2 \%$ chlorhexidine solution for the prevention of ventilator-associated pneumonia. Infect Control Hosp Epidemiol 2008;29(2):131-136.

15. Bellissimo-Rodrigues F, Bellissimo-Rodrigues WT, Viana JM, Teixeira GC, Nicolini E, Auxiliadora-Martins M, et al. Effectiveness of oral rinse with chlorhexidine in preventing nosocomial respiratory tract infections among intensive care unit patients. Infect Control Hosp Epidemiol 2009;30(10):952-958.

16. Munro CL, Grap MJ, Jones DJ, McClish DK, Sessler CN. Chlorhexidine, toothbrushing, and preventing ventilator-associated pneumonia in critically ill adults. Am J Crit Care 2009;18(5):428-437.

17. Scannapieco FA, Yu J, Raghavendran K, Vacanti A, Owens SI, Wood K, Mylotte JM. A randomized trial of chlorhexidine gluconate on oral bacterial pathogens in mechanically ventilated patients. Crit Care 2009;13(4):R117.

18. Cabov T, Macan D, Husedzinović I, Skrlin-Subić J, Bosnjak D, Sestan-Crnek S. The impact of oral health and $0.2 \%$ chlorhexidine oral gel on the prevalence of nosocomial infections in surgical intensive-care patients: a randomized placebo-controlled study. Wien Klin Wochenschr 2010;122(13-14):397-404.

19. Berry AM, Davidson PM, Masters J, Rolls K, Ollerton R. Effects of three approaches to standardized oral hygiene to reduce bacterial colonization and ventilator associated pneumonia in mechanically ventilated patients: a randomised control trial. Int J Nurs Stud 2011; 48(6):681-688.

20. Grap MJ, Munro CL, Hamilton VA, Elswick RK Jr., Sessler CN, Ward KR. Early, single chlorhexidine application reduces ventilatorassociated pneumonia in trauma patients. Heart Lung 2011;40(5): e115-e122.

21. Jácomo AD, Carmona F, Matsuno AK, Manso PH, Carlotti AP. Effect of oral hygiene with $0.12 \%$ chlorhexidine gluconate on the incidence of nosocomial pneumonia in children undergoing cardiac surgery. Infect Control Hosp Epidemiol 2011;32(6):591-596.

22. Kusahara DM, Peterlini MA, Pedreira ML. Oral care with $0.12 \%$ chlorhexidine for the prevention of ventilator-associated pneumonia in critically ill children: randomised, controlled and double blind trial. Int J Nurs Stud 2012;49(11):1354-1363.

23. Meinberg MC, Cheade Mde F, Miranda AL, Fachini MM, Lobo SM. The use of $2 \%$ chlorhexidine gel and toothbrushing for oral hygiene of patients receiving mechanical ventilation: effects on ventilatorassociated pneumonia. Rev Bras Ter Intensiva 2012;24(4):369-374.

24. Özçaka Ö, Basoğlu OK, Buduneli N, Taşbakan MS, Bacakoğlu F, Kinane DF. Chlorhexidine decreases the risk of ventilator-associated pneumonia in intensive care unit patients: a randomized clinical trial. J Periodontal Res 2012;47(5):584-592. 


\section{INTRAoral ChlorheXidine For PreVEnTION OF VAP}

25. Sebastian MR, Lodha R, Kapil A, Kabra SK. Oral mucosal decontamination with chlorhexidine for the prevention of ventilator-associated pneumonia in children: a randomized, controlled trial. Pediatr Crit Care Med 2012;13(5):e305-e310.

26. Labeau SO, Van de Vyver K, Brusselaers N, Vogelaers D, Blot SI. Prevention of ventilator-associated pneumonia with oral antiseptics: a systematic review and meta-analysis. Lancet Infect Dis 2011;11(11): 845-854.

27. Li J, Xie D, Li A, Yue J. Oral topical decontamination for preventing ventilator-associated pneumonia: a systematic review and meta-analysis of randomized controlled trials. J Hosp Infect 2013;84(4):283-293.

28. Shi Z, Xie H, Wang P, Zhang Q, Wu Y, Chen E, et al. Oral hygiene care for critically ill patients to prevent ventilator-associated pneumonia. Cochrane Database Syst Rev 2013;(8):CD008367.

29. Klompas M, Speck K, Howell MD, Greene LR, Berenholtz SM. Reappraisal of routine oral care with chlorhexidine gluconate for patients receiving mechanical ventilation: systematic review and meta-analysis. JAMA Intern Med 2014;174(5):751-761.

30. Zhang TT, Tang SS, Fu LJ. The effectiveness of different concentrations of chlorhexidine for prevention of ventilator-associated pneumonia: a meta-analysis. J Clin Nurs 2014;23(11-12):1461-1475.

31. Liberati A, Altman DG, Tetzlaff J, Mulrow C, Gøtzsche PC, Ioannidis JP, et al. The PRISMA statement for reporting systematic reviews and meta-analyses of studies that evaluate healthcare interventions: explanation and elaboration. BMJ 2009;339:b2700.

32. Clarke M OA, Paulsen E, Higgins JPT, Green S. Assessing risk of bias in included studies. In: Higgins JPT and Green S, editors. Cochrane Handbook for Systematic Reviews of Interventions Ver- sion 5.1.0. The Cochrane Collaboration; 2011. www.cochranehandbook.org. Accessed April 13, 2016.

33. Firth MA, Shewen PE, Hodgins DC. Passive and active components of neonatal innate immune defenses. Anim Health Res Rev 2005; 6(2):143-158.

34. Martin TR, Ruzinski JT, Wilson CB, Skerrett SJ. Effects of endotoxin in the lungs of neonatal rats: age-dependent impairment of the inflammatory response. J Infect Dis 1995;171(1):134-144.

35. Siegrist $C A$. Vaccination in the neonatal period and early infancy. Int Rev Immunol 2000;19(2-3):195-219.

36. Snyders O, Khondowe O, Bell J. Oral chlorhexidine in the prevention of ventilator-associated pneumonia in critically ill adults in the ICU: a systematic review. Southern African J Crit Care 2011;27(2): 48-56.

37. Addy M, Jenkins S, Newcombe R. The effect of some chlorhexidinecontaining mouthrinses on salivary bacterial counts. J Clin Periodontol 1991;18(2):90-93.

38. Harper PR, Milsom S, Wade W, Addy M, Moran J, Newcombe RG. An approach to efficacy screening of mouthrinses: studies on a group of French products (II): inhibition of salivary bacteria and plaque in vivo. J Clin Periodontol 1995;22(9):723-727.

39. White RR, Hays GL, Janer LR. Residual antimicrobial activity after canal irrigation with chlorhexidine. J Endod 1997;23(4):229-231.

40. Scannapieco FA, Bush RB, Paju S. Associations between periodontal disease and risk for nosocomial bacterial pneumonia and chronic obstructive pulmonary disease: a systematic review. Ann Periodontol 2003;8(1):54-69. 